\title{
A good beginning makes a good ending: association between acute pain trajectory and chronic postsurgical pain
}

\author{
Fumimasa Amaya ${ }^{1}$ (D)
}

Received: 7 October 2018 / Accepted: 18 October 2018 / Published online: 26 October 2018

(c) Japanese Society of Anesthesiologists 2018

Chronic pain continuing for a long time after surgery is one of the emerging medical problems in the field of pain medicine. The presence of chronic postsurgical pain is associated with poor health-related quality of life, increased medical costs and negative psychological effects [1]. In the United States, $5 \%$ of opioid naive patients become long-term opioid users after surgery [2].

Chronic postsurgical pain was initially defined as pain continuing for at least 2 months [3]. However, it was debated that 2 months might be too short a period to completely exclude the "functional results of the surgical procedure" [4]. The more recently developed classification of chronic pain according to the International Classification of Diseases 11th Revision (ICD-11) defines chronic postsurgical pain as pain that persists for at least 3 months after the surgery [5]. Based on this definition, the incidence of chronic postsurgical pain was estimated as $10-50 \%$ [6]. Although its frequency varies depending on the type of surgery, the degree of tissue injury or presence of nerve injury does not always correlate with the incidence of chronic postsurgical pain $[7,8]$. The high prevalence of chronic postsurgical pain is a global phenomenon. Sugiyama et al. conducted a multicenter survey in Japan to investigate the prevalence of chronic postsurgical pain after total knee arthroplasty (TKA) and lung cancer surgery, and concluded that chronic pain prevalence was $49 \%$ and $18 \%$ at 3 months after TKA and lung surgery, respectively [9].

Chronic postsurgical pain has unique characteristics based on the event that leads to the chronic pain condition. Most patients undergoing elective surgery have a chance to receive preventive analgesic intervention. Consequently, several studies have been conducted to determine perioperative

Fumimasa Amaya

ama@koto.kpu-m.ac.jp

1 Department of Pain Management and Palliative Care Medicine, Kyoto Prefectural University of Medicine, Kajii-cho 465, Kamigyo-ku, Kyoto 6028566, Japan analgesic interventions to prevent postsurgical pain. Unfortunately, most of the interventions studied were either ineffective or effective in only a particular clinical setting [10]. The best alternative so far is to identify high-risk patients soon after surgery, and provide a pain management plan aimed to minimize chronic pain and its negative impact on quality of life [11]. It is crucial, therefore, to identify patients at risk of developing chronic postsurgical pain soon after the surgery [10].

It is important to remember the simple fact that all chronic postsurgical pains begin as acute pain. As I mentioned above, we have to wait for 3 months to make a definitive diagnosis of chronic postsurgical pain, yet we may be able to find an earlier postoperative period during which the pain phenotype enables us to distinguish the subclass of patients who are at high risk for developing chronic postsurgical pain in the future.

Ample studies have demonstrated that the intensity of acute postsurgical pain (mostly recorded on day 0 or day 1 ) is associated with chronic postsurgical pain [12]. However, pain intensity on day 1 may not be an adequate measure of the magnitude of postsurgical pain the patient experiences during the acute phase. Based on our clinical experience, we know that the intensity of acute postsurgical pain changes on a daily basis toward its resolution. Development of trajectory analysis of daily pain intensity allows us to determine the longitudinal characteristics of acute pain after the surgery [13]. There is considerable inter-individual variability in initial pain intensity and rate of resolution [14], suggesting that all postsurgical patients do not always follow the same trajectory of acute pain after the surgery.

Lavand'homme et al. demonstrated that patients who will develop chronic postsurgical pain in the future have a distinctive acute pain trajectory that is different from that in patients who will not develop chronic postsurgical pain [15]. This "backward" approach allowed us to hypothesize that there may be several acute pain trajectories, and each trajectory has distinctive characteristics in terms of initial pain 
intensity and rate of resolution, as well as different risks of development of chronic postsurgical pain. By finding clusters of acute pain trajectories and analyzing their association with chronic pain, we may be able to distinguish patients at high risk for chronic postsurgical pain earlier.

A group-based trajectory model (GBTM) enables clustering of patients who follow a similar trajectory of longitudinal data [16]. Using GBTM analysis, Okamoto et al. developed three cluster trajectories of acute pain for 7 days after breast cancer surgery [17]. Among the three (mild, moderate and severe pain) clusters, patients with a severe pain trajectory turned out to have a high risk of chronic pain 6 months after the surgery. A similar approach has been attempted following total hip arthroplasty, by evaluating a GBTM-based acute pain trajectory for 5 days after the surgery, although it failed to predict future chronic pain [18]. It seems that information on pain intensity for approximately 7 days after surgery has substantially contributed to the accuracy of prediction of the likelihood of development of chronic postsurgical pain. Combined analysis of three prospective studies found that pain intensity on day 7 is an important factor to predict chronic pain after breast cancer surgery [19].

Several factors are suggested to contribute to the late recovery of acute postsurgical pain. Clinical evidence identified several preoperative patient characteristics (pain prior to the surgery, pain expectation, etc.) as risk factors for long-lasting intense pain after the surgery [20]. Animal studies suggested that the duration of pain hypersensitivity after the tissue incision is prolonged by factors during the surgery (remifentanil infusion [21]) or after the surgery (slight inflammation at the site of injury [22]). We need to understand individual stories of acute pain trajectories leading to chronic postsurgical pain to determine the best available treatment options for each patient. Integration of these stories will shed light on the novel mechanisms of transition from acute to chronic pain.

\section{Compliance with ethical standards}

Conflict of interest The authors have no conflict of interest with this article.

\section{References}

1. Rashiq S, Dick BD. Post-surgical pain syndromes: a review for the non-pain specialist. Can J Anaesth. 2014;61:123-30.

2. Brummett CM, Waljee JF, Goesling J, Moser S, Lin P, Englesbe MJ, Bohnert AS, Kheterpal S, Nallamothu BK. New persistent opioid use after minor and major surgical procedures in US adults. JAMA Surg. 2017: e170504.

3. Macrae WA. Chronic post-surgical pain: 10 years on. $\mathrm{Br} \mathbf{J}$ Anaesth. 2008;101:77-86.
4. Werner MU, Kongsgaard UE. I. Defining persistent post-surgical pain: is an update required? Br J Anaesth. 2014;113:1-4.

5. Treede RD, Rief W, Barke A, Aziz Q, Bennett MI, Benoliel R, Cohen M, Evers S, Finnerup NB, First MB, Giamberardino MA, Kaasa S, Kosek E, Lavand'homme P, Nicholas M, Perrot S, Scholz J, Schug S, Smith BH, Svensson P, Vlaeyen JW, Wang SJ. A classification of chronic pain for ICD-11. Pain. 2015;156:1003-7.

6. Kehlet H, Jensen TS, Woolf CJ. Persistent postsurgical pain: risk factors and prevention. Lancet. 2006;367:1618-25.

7. Fuzier R, Puel F, Izard P, Sommet A, Pierre S. Prospective cohort study assessing chronic pain in patients following minor surgery for breast cancer. J Anesth. 2017;31:246-54.

8. Jaaskelainen SK, Teerijoki-Oksa T, Virtanen A, Tenovuo O, Forssell $\mathrm{H}$. Sensory regeneration following intraoperatively verified trigeminal nerve injury. Neurology. 2004;62:1951-7.

9. Sugiyama Y, Iida H, Amaya F, Matsuo K, Matsuoka Y, Kojima K, Matsuno F, Hamaguchi T, Iseki M, Yamaguchi K, Takahashi Y, Hara A, Sugasawa Y, Kawamata M, Tanaka S, Inagaki Y, Otsuki A, Yamazaki M, Ito H. Prevalence of chronic postsurgical pain after thoracotomy and total knee arthroplasty: a retrospective multicenter study in Japan (Japanese Study Group of Subacute Postoperative Pain). J Anesth. 2018;32:434-8.

10. Tawfic Q, Kumar K, Pirani Z, Armstrong K. Prevention of chronic post-surgical pain: the importance of early identification of risk factors. J Anesth. 2017;31:424-31.

11. Katz J, Weinrib A, Fashler SR, Katznelzon R, Shah BR, Ladak SS, Jiang J, Li Q, McMillan K, Santa Mina D, Wentlandt K, McRae K, Tamir D, Lyn S, de Perrot M, Rao V, Grant D, RocheNagle G, Cleary SP, Hofer SO, Gilbert R, Wijeysundera D, Ritvo P, Janmohamed T, O'Leary G, Clarke H. The Toronto General Hospital Transitional Pain Service: development and implementation of a multidisciplinary program to prevent chronic postsurgical pain. J Pain Res. 2015;8:695-702.

12. Perkins FM, Kehlet H. Chronic pain as an outcome of surgery. A review of predictive factors. Anesthesiology. 2000;93:1123-33.

13. Chapman CR, Donaldson GW, Davis JJ, Bradshaw DH. Improving individual measurement of postoperative pain: the pain trajectory. J Pain. 2011;12:257-62.

14. Tighe PJ, Le-Wendling LT, Patel A, Zou B, Fillingim RB. Clinically derived early postoperative pain trajectories differ by age, sex, and type of surgery. Pain. 2015;156:609-17.

15. Lavand'homme PM, Grosu I, France MN, Thienpont E. Pain trajectories identify patients at risk of persistent pain after knee arthroplasty: an observational study. Clin Orthop Relat Res. 2014;472:1409-15.

16. Nagin DS, Odgers CL. Group-based trajectory modeling in clinical research. Annu Rev Clin Psychol. 2010;6:109-38.

17. Okamoto A, Yamasaki M, Yokota I, Mori M, Matsuda M, Yamaguchi Y, Yamakita S, Ueno H, Sawa T, Taguchi T, Hosokawa $\mathrm{T}$, Amaya F. Classification of acute pain trajectory after breast cancer surgery identifies patients at risk for persistent pain: a prospective observational study. J Pain Res. 2018;11:2197-206.

18. Page MG, Katz J, Curtis K, Lutzky-Cohen N, Escobar EM, Clarke HA. Acute pain trajectories and the persistence of postsurgical pain: a longitudinal study after total hip arthroplasty. J Anesth. 2016;30:568-77.

19. Meretoja TJ, Andersen KG, Bruce J, Haasio L, Sipila R, Scott NW, Ripatti S, Kehlet H, Kalso E. Clinical prediction model and tool for assessing risk of persistent pain after breast cancer surgery. J Clin Oncol. 2017;35:1660-7.

20. Sipila RM, Haasio L, Meretoja TJ, Ripatti S, Estlander AM, Kalso EA. Does expecting more pain make it more intense? Factors associated with the first week pain trajectories after breast cancer surgery. Pain. 2017;158:922-30. 
21. Celerier E, Gonzalez JR, Maldonado R, Cabanero D, Puig MM. Opioid-induced hyperalgesia in a murine model of postoperative pain: role of nitric oxide generated from the inducible nitric oxide synthase. Anesthesiology. 2006;104:546-55.

22. Matsuda M, Oh-Hashi K, Yokota I, Sawa T, Amaya F. Acquired exchange protein directly activated by cyclic adenosine monophosphate activity induced by p38 mitogen-activated protein kinase in primary afferent neurons contributes to sustaining postincisional nociception. Anesthesiology. 2017;126:150-62. 